\title{
Agent-based Market Research Learning Environment for New Entrepreneurs
}

Recibido:

Julio 30 de 2011

Aceptado:

Marzo 4 de 2012

\section{Abstract}

Due to the importance of creating alternative mechanisms to generate know-how on potential markets for new entrepreneurs this paper proposes an agent-based learning environment to help them learning market research strategies within new businesses. An instructor agent, serving as a learning assistant within the MAS environment guides new entrepreneurs to identify their most adequate market niche. The integration of MAS-CommonKADS and GAIA methodologies is used along with AUML diagrams in order to design and develop this agentbased learning environment, called MaREMAS. The paper thus describes all the stages concerning MaREMAS construction focusing on the conceptualization, analysis, design, prototype development, and validation. The tests developed in the MaREMAS learning environment were satisfactory, however, it is proposed as future work to provide the system a more robust statistical module that allows a better analysis of the research variables and hence be able to generate more useful suggestions to the entrepreneur.

Keywords: Agent-based learning environments, market research strategies, entrepreneurship, intelligent learning systems, learning helpers modeling

\section{Introduction}

$\mathrm{M}$ any researchers have found that entrepreneurship can contribute to economic growth acting as a method to discover and generate knowledge based on the market opportunities recognition and trading [1]. It is thought that an efficient management of the business creation process becomes a filter to guide towards the selection of successful enterprises within a given market [2]. Thus, training and learning strategies to exploiting market opportunities are adequate means to improve the skills and abilities of new companies.
Universidad Nacional de Colombia - Sede Medellín Facultad de Minas javalenca@unal.edu.co omsalazaro@unal.edu.co dovalle@unal.edu.co gawad@unal.edu.co 


\section{Respuestas}

Año 17

No. 1

Junio 2012

ISSN 0122-820X
Alternative mechanisms to generate intelligence should be searched in terms of competition and potential markets [3], using generative learning strategies [4], moreover, specific tools to integrate the breadth of knowledge spread on the market are required. The utilization of experts and consultants for integrating knowledge related to environmental assessments is one of the most used mechanisms [5].

Performing a traditional market research and recruitment consultancy is a process that can be costly in time and resources for new entrepreneurs. The existence of an opportunity not only depends on the presence of a market with a threshold of customers, depends on the creative and innovative capacity of the company to generate a higher market value compared with competitors [3]. Market research aims to help the new enterprises in their learning and decision making to determine the existing market for their product or service, facilitating the identification of a specific market niche [6]. Consequently, the relationship between entrepreneurship and market research allows the value creation and the resources acquisition in order to capitalize on market opportunities. The development of systems serving as instructors in marketing research for new entrepreneurs is an alternative that allows a better use of resources, a broader knowledge of the market and stimulates creative activity in the discovery of new potential expansion niche markets.

Our agent-based learning environment proposed for Market Research Learning purposes aims to be an instructor in the marketing research process established by McDaniel and Gates [8] which specifies standard phases of academic literature [4], [5]. Figure 1 shows all the steps involved in the learning process market research.

The ultimate goal is that the user understands and learns about the stages and components of the market research process, thus, it is proposed a sequential process that allows it to feedback in each of the learning process steps, i.e., a process that serves as guideline to make a proper market research and promote creativity and pro-activity regarding the opportunities offered by the business environment. A Multi-Agent System learning (MAS) environment can provide support in the strategies of market orientation that are compatible with the generation of business intelligence, improving standards of reliability to create the company, and also, filling some gaps that new businessmen have in the marketing orientation due to their limited resources [3].

Fig. 1. Components of the market research process (Source: [8])

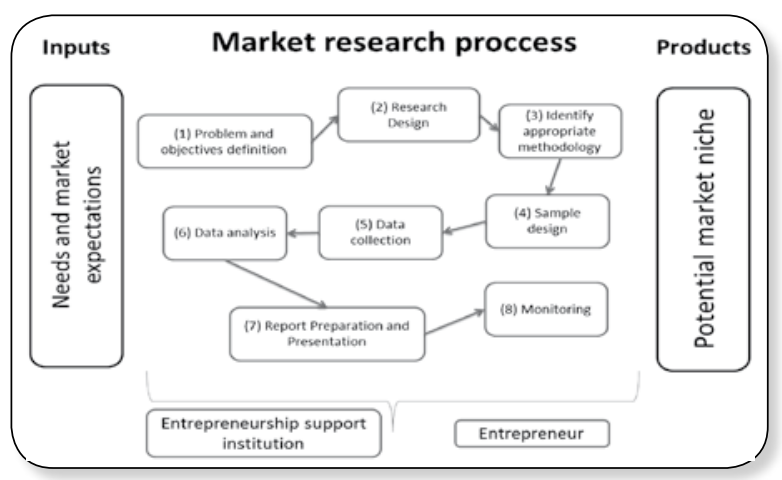

The rest of the paper is organized as follows: Section 2 outlines main concepts on MAS development methodologies involved in this research. Section 3 describes Modeling, Implementation and Validation of MaREMAS. Section 4 offers main results of system's validation. Finally, conclusions and future work are presented in Section 5.

\section{Methodology}

The first thing that arises when developing a MAS is the selection of an appropriate conceptualization, analysis, and design process methodology. The decision is complex due to the fact of the existence of a variety of methodologies such as MaSE (Multiagent System Engineering) [8], MASCommonKADS [9], GAIA [10], among others. It is important to highlight that a single 
methodology is not sufficiently complete to reflect the flexibility, interaction among agents and the complexity of the organizational multi-agent system structures. MASCommonKADS offers several advantages about the proposal of models development [11] which reflect different perspectives and of the MAS proposed.

Fig. 2. Match between MAS-CommonKADS's models and GAIA's models (Source: [12])

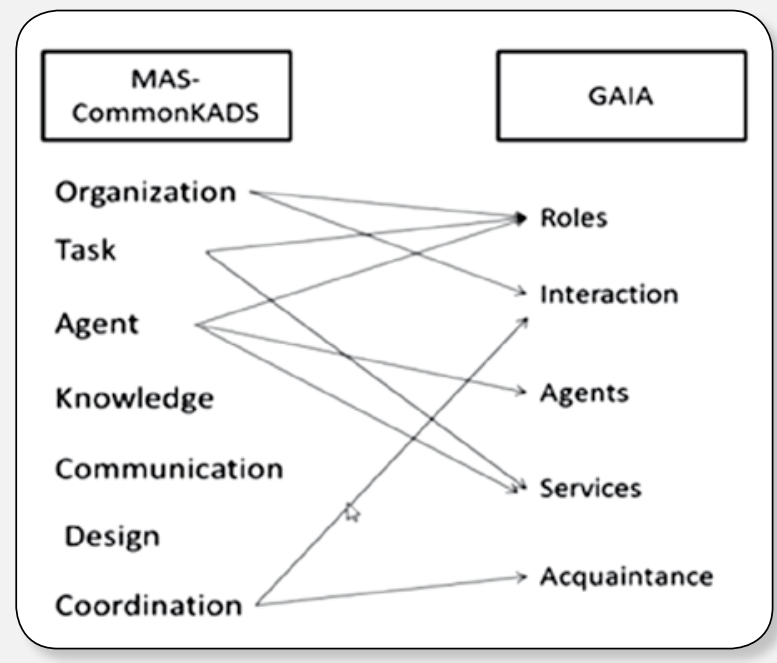

The MAS-CommonKADS methodology provides a framework for the specification of implementation-independent knowledge, combining a set of reusable knowledge models used by frequently performed tasks. GAIA models were used to enrich the system modeling. These models do not impose restrictions on the implementation and give to the developer the ability of choosing the desired structure [11]. The MASCommonKADS and GAIA methodologies were complementary each other in different ways: the GAIA methodology uses an iterative process at each stage of the system development while MAS-CommonKADS approach uses a cyclical process that allows that the analysis and design phases can perform in an evolutionary manner, complementing the lack of tracing and tracking errors and risks attending in GAIA [10]. Both methodologies provide a set of models to be used during the MAS development. In addition, although all the models represent different issues of the system they have in common that they offer a description of the agent's environment, and the relationship and dependencies among agents. Figure 2 shows the main equivalences among models of MAS-CommonKADS and GAIA methodologies.

The integration of both methodologies facilitates the MAS design process giving the system developers with more flexibility to make their own decisions. In fact, the representation of all the aspects of the MAS based on models implies a better understanding by developers and actors providing the possibility of system upgrades in the short and long term.

MAS-CommonKADS and GAIA methodologies are used along with AUML diagrams [8], [9], which is not a methodology but instead focuses on trying to adapt existing development tools in order to enrich agentbased system modeling [12]. The joint application of these methodologies will be described throughout the paper where the models are developed using textual templates and diagrams that explain the structure of the main instructor agent (researcher agent). The MAS raised in this paper is called MaREMAS (Market Research for Entrepreneurs based on a Multi-Agent System Approach).

\section{MaREMAS: Modeling, Implementation and Validation}

\subsection{First Phase: MAS Conceptualization}

First of all, the identification and description of actors and their related use cases are required when using the MASCommonKADS methodology [9] based on the conceptualization phase definitions as a first approach to MaREMAS. This kind of analysis is user-centered, considering the points of view regarding the user requirements 
and own needs of the system. The use case technique allows the developer to obtain the user requirements and generate test cases [9]. In MaREMAS the actors identified are: entrepreneur, entrepreneurship support institution, researcher, and technical marketing. Figure 3 illustrates the case where the researcher (instructor) interferes and interacts with the system.

Fig. 3. Use case diagram for the market research process

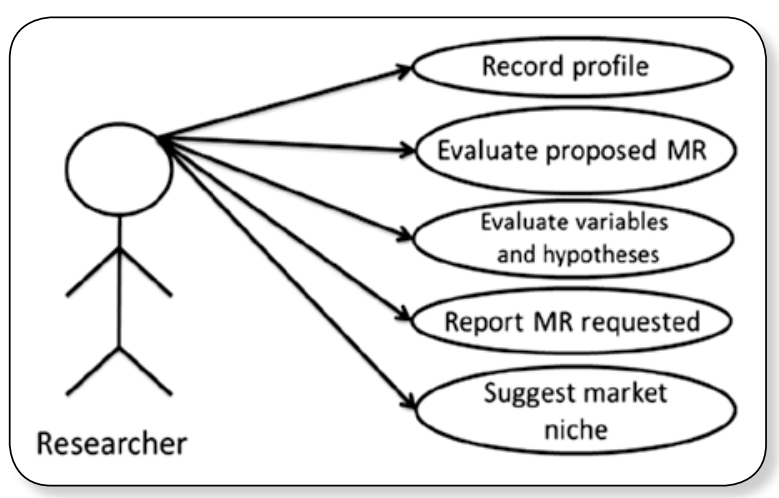

The identification of use cases, conditions, descriptions, and applications are de-signed based on the process that must be considered for the development of a market research.

\subsection{Second Phase: MAS Analysis}

The initial diagrams to analyze MaREMAS are handled by GAIA methodology, which provides the agent system organization. This organization refers to a set of roles which keep certain relationships among agents, making clear the hierarchy of analysis concepts that lead with the process. GAIA defines a system as a set of organized roles and interactions among them, i.e., an agent-based society [10].

- Role Model. MaREMAS presents the role of each entity within the system, identifies four roles that correspond to the four actors in the use case model. The actors identified are the following: entrepreneur, entrepreneurship support institution, researcher, and technical marketing assistant, who interact among them through relations of communication/collaboration. Each role in the system must be defined through main issues such as objectives, responsibilities, skills, and role's information required.

Fig. 4. Researcher agent reasoning mechanism

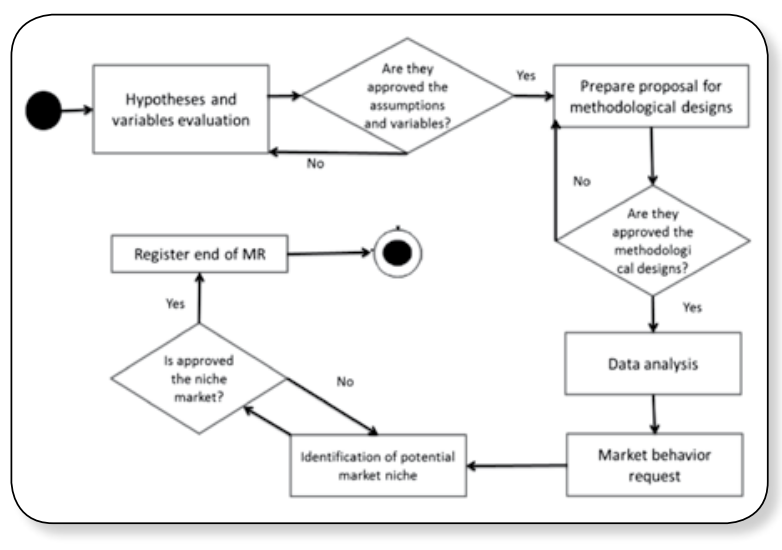

- Agent Model. The purpose of this model is documenting the different types of agents to be used in the system [9], defining the instances number to be had from everyone in the runtime. An agent type is a set of roles, i.e., a type of agent may take one or more roles but the opposite isn't true [10]. For efficiency or design reasons, several roles can be grouped into the same agent [13]. To identify the roles is necessary to analyze the system interactions and the actor capacity to play multiple roles. From roles previously defined the following 4 agents were considered: Entrepreneur, Support Institution, Operator, and Researcher.

- Task Model. This model describes the tasks that agents can perform: objectives of each task, its components and problem solving methods [9]. The decomposition of tasks into sub-tasks for MaREMAS is related to the Market Research (MR) for new venture creation that mainly implies the following three subtasks: Request MR, Design MR, and Analyze the MR.

- Experience or Knowledge Model. The experience model is adapted from MASCommonKADS; this describes the knowledge needed by agents to achieve their objectives [13]. Figure 4 shows the inference mechanism used as the researcher agent reasoning proposed in MaREMAS. 
- Coordination Model. The coordination model specifies the interactions among agents involved in the problem solving. This model is used to store decisions on the communication protocol structure, along with the risks associated with the functional requirements of communication protocols [9]. Figure 5 shows the MaREMAS's AUML activity diagram allows observing the agents interaction protocols, expressing the activities and operations carried out and the facts that cause them. In addition, it provides a useful explicit control thread for complex interaction protocols that involves simultaneous processing.

- Communication Model. In MASCommonKADS communication model is responsible for modeling human-machine relationships [9].

- Organization Model. The MASCommonKADS organization model aims to analyze, from a group perspective, the structural relationships among agents (software and humans) that interact within the system. This model represents the organization in terms of related suborganizations [9] to better understand the general system structure. The MaREMAS's organization exhibits the existence of few hierarchies; there is only one between the researcher and the operator (marketing technician). The other agents have horizontal relationships and cooperate to carry out the proposed objectives.

Fig. 5. AUML activity diagram for market research protocol

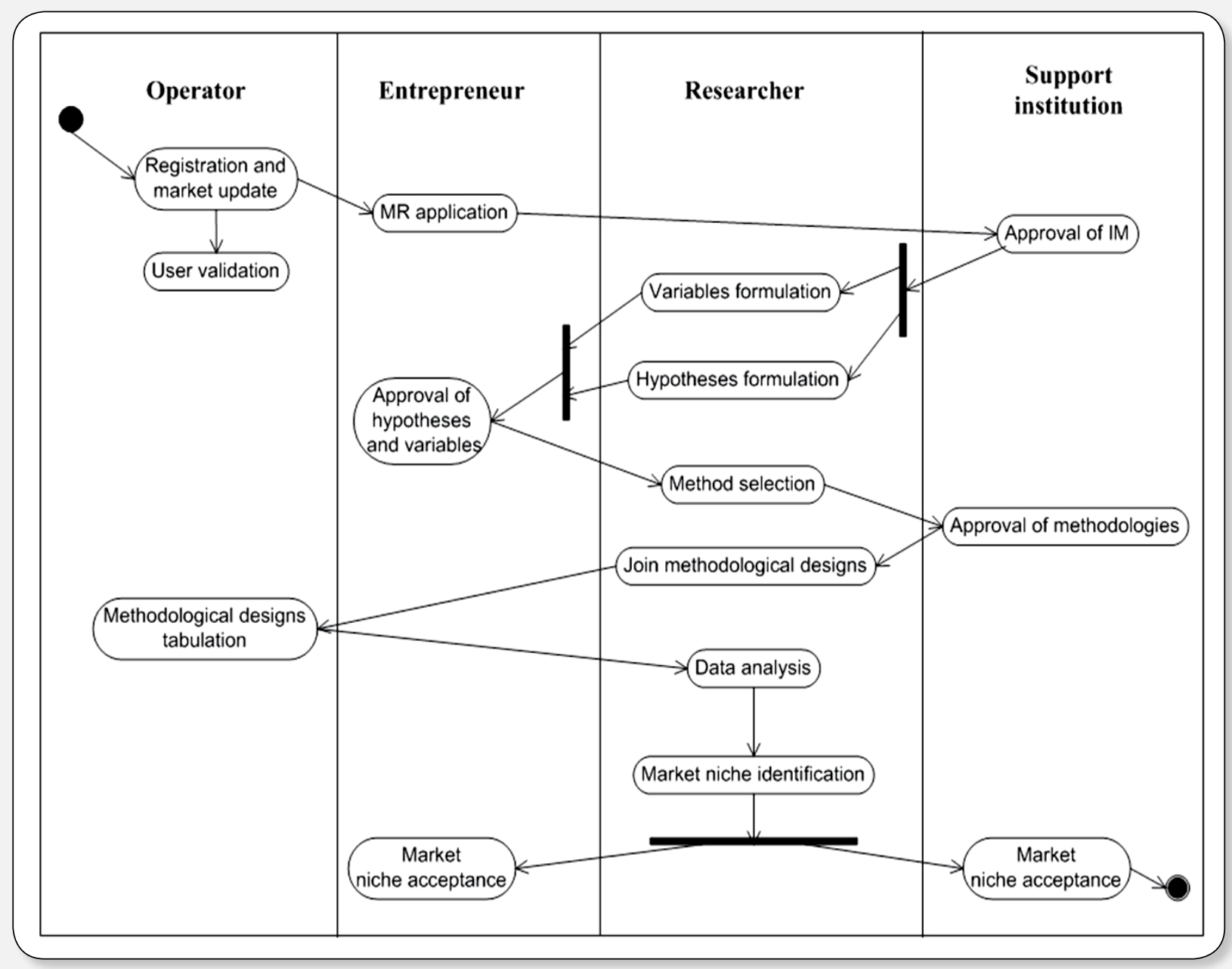




\subsection{Third Phase: MAS Design}

- Service Model. The service concept implies that in a MAS designed with GAIA the agents can perform tasks that are offered to other agents when they required [10]. This model aims to identify and specify those services, associated with each agent role, that represent its functionality. Thus, each activity previously identified in the analysis phase will correspond to a service, although not all services necessarily emerge from activities. The method proposed by Bustos [14] and used in MaREMAS, consists of a template that specifies: service name, input data (what is sent), output data (what is obtained) and pre-conditions (conditions that must be satisfied before the service starts). The main services implemented in MaREMAS are the following: Request_MR, Generate_ variables, Generate_hypothesis, Suggest_ methodologies, Elaborate_budget, Analyze_data, Identify_market_niche, Register_user, Enter_market_segment_information, among others.

-Design Model. TheMAS-CommonKADS design model documents the design decisions adopted [7]. Thismodel specifies the functional requirements of the MAS, introduce the non-functional requirements that should be considered for its implementation and technical aspects that were not previously captured. Three kinds of design decisions should be made: network design (outlines the network model), agents design (each agent decomposes into subsystems), and platform design (software and hardware aspects) [9]. Figure 6 shows MaREMAS components, their location, and communication channels among modules and the connection with other systems and information repositories. In the AUML component diagram it can be seen three different modules, the first is the host_ 1 wherein resides the JADE central platform with its main container composed of specific JADE agents and MaREMAS agents interacting with the MR process.

The other two modules represent different secondary JADE containers; each associated with entrepreneurship support institutions and having several researcher agents. All hosts from 2 to $\mathrm{k}$ it will have the ability to communicate with host 1 using the RMI protocol provided by JAD̄E to call remotely their agents and functions. The operator agent stores in a DB history the MR requested,

Fig. 6. MaREMAS Component diagram

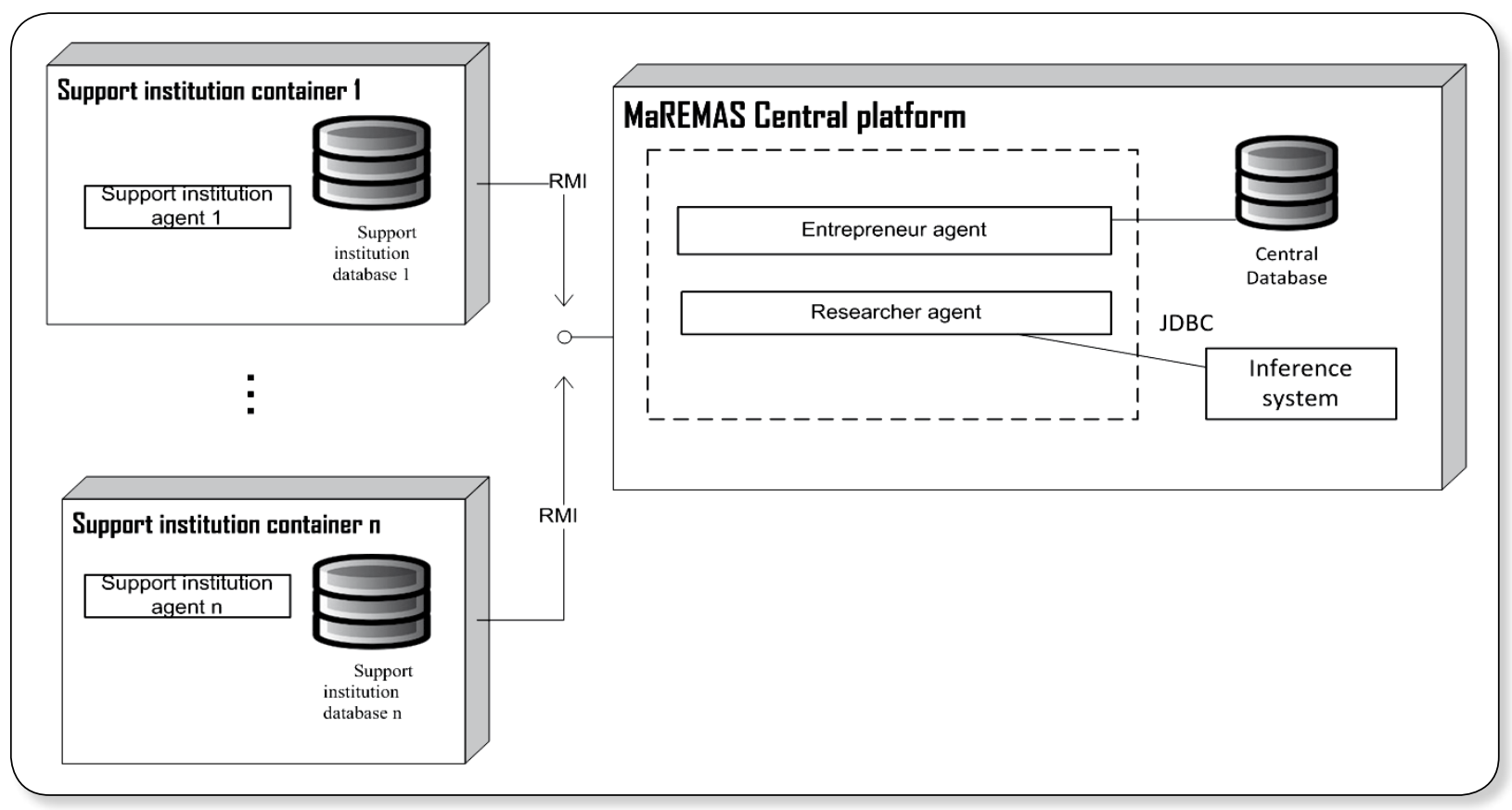


its variables, assumptions, and results to consolidate more market information generating useful information feedback for the MAS that will provide increasingly successful recommendations about market niche. The operator agent and researcher agent have access to the system DB through JDBC compliant with the DBMS used.

Fig. 7. JADE RMA interface for the MAS

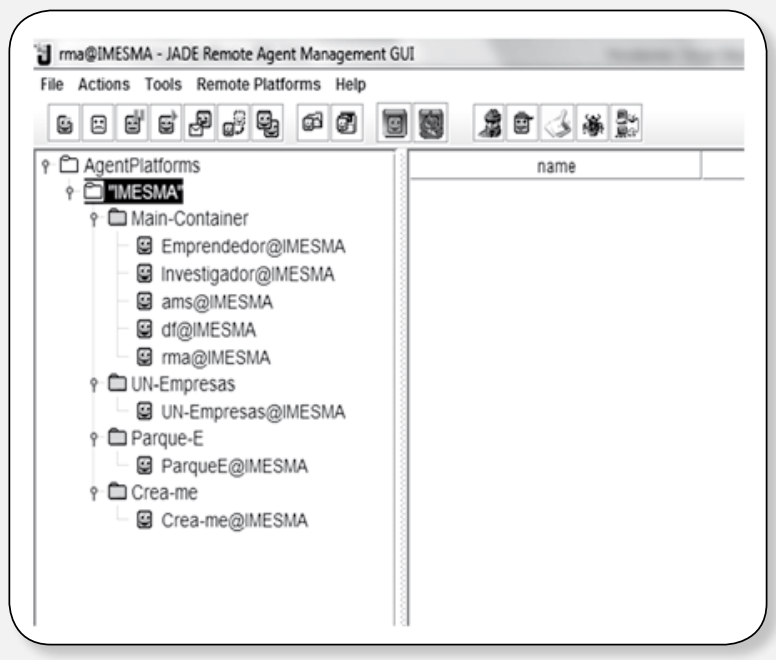

Fig. 8. MR Interface design

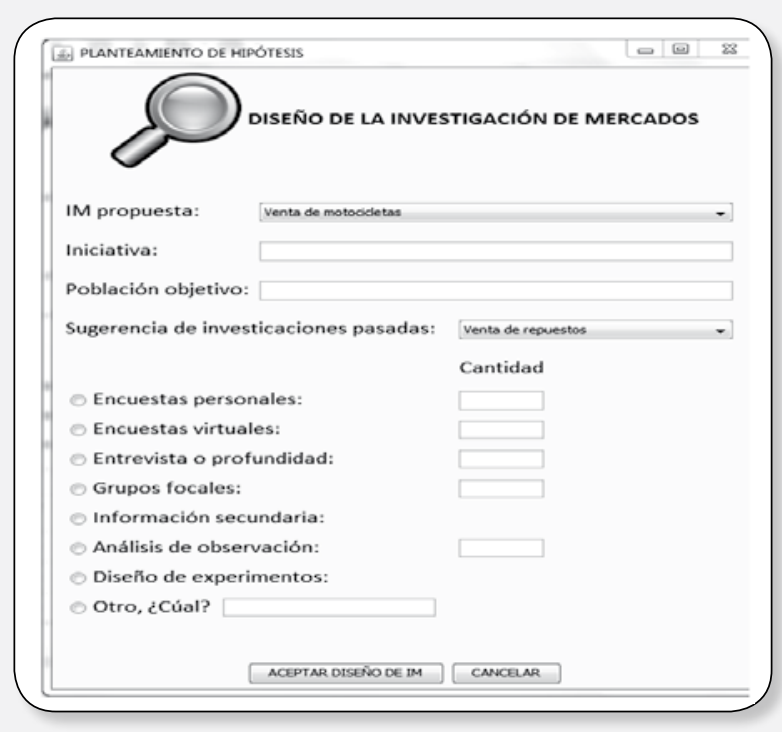

The platform design documents low-level decisions on the implementation language selected; in this case MaREMAS, JADE FIPA-compliant ("JAVA Agent Developmet Framework") [15],[16] was used along with MySQL Server 5.0 as DBMS, which allows relational databases creation, multi-threaded, and multi-users to exchange information among agents making possible to real-time information transfer. Another tool used to develop the system was hibernate 3.6.4 which allows the object/relational mapping (ORM) for the Java platform, providing transactions transparent and efficient with the DB.

\subsection{Prototype Implementation and Validation}

Figures 7 and 8 show some of the system interfaces, which incorporate the functional and non-functional requirements found in the analysis phase. In addition, the registration stage offers a wizard that allows the user to enter research parameters, providing a friendly interface and providing support throughout the process of filling out the necessary information. The system offers a teachinglearning module which allows the user to enter new MR results or otherwise offers him earlier research related to the parameters entered into the record business idea.

\section{Results}

A main feature offered by MaREMAS is the ability to make decisions in real time according to the parameters entered by the entrepreneur. The main decisions taken by MaREMAS are the following: (a) Evaluates and suggests MR variables; (b) Evaluates and suggests MR hypotheses; (c) Evaluates and suggests methodological designs; (d) Evaluate the budget and (e) Suggests potential market niches based on the MR results.

In addition, the researcher agent promotes the acquisition of MR life cycle learning strategies in the user since it helps to identify the variables and assumptions involved in the $\mathrm{MR}$ and provides suggestions recovered from the MR database. This allows the entrepreneur to consider areas of study and problems that had not previously been considered. The system also provides guidance to users for generating ideas and initiatives in terms of potential customers (market) to be consulted. 
The sequence of the main interactions between the entrepreneur user and the researcher agent initially occurs when the entrepreneur enters the variables to analyze along with the MR business sector desired. With this information the researcher agent generates variable suggestions or in case of MaREMAS has previously adequate research information on the subject stored this will be presented. Consequently, the entrepreneur formulates hypotheses based on the variables analyzed and the researcher agent evaluates if the hypotheses are correctly formulated and if they accomplish the minimum number required. Finally, the researcher agent suggests new combinations of variables to generate new hypotheses for study.

In order to evaluate the system's performance we decided to consider two kinds of scenarios: The first one was related to the proposition of a new MR that was not stored in the MaREMAS's knowledge base. This scenario made it possible to assess how efficient was the system as an instructor of new entrepreneurs, guiding them in the MR designing process. In addition, the system provides relevant data useful for the MR such as variables, hypotheses, appropriate methodological design, budget, among others. For the second scenario, it was requested MR related to studies previously entered to MaREMAS. This scenario got a satisfactory answer from MaREMAS since when a MR was requested, the system offered very well suggestions with respect to the unit analysis, covered in the MR.

From the results obtained by the tests it can be noted that decisions made by software agents are coherent to those taken by human agents. In addition, the system offers benefits in terms of response time compared with support institutions when they may advise to new entrepreneurs about MR processes. Finally, there is a module in MaREMAS that offers suggestions on previous MR studies, which expedites the learning process and data gathering, thus saving resources of time and budget.

\section{Conclusions and Future Work}

The integration of MAS-CommonKADS and GAIA methodologies along with the assistance of AUML diagrams provides more flexibility in the agents interaction and the complexity of the MAS organizational structures. In fact, the representation of all the aspects of the MAS based on models provided by both methodologies implies a better understanding by developers and actors giving the possibility of system upgrades in the short and long term. This paper showed in detail the design and development cycle of MaREMAS, an agent-based learning environment that serves as instructor for new entrepreneurs, teaching and guiding them on the MR process. The process includes from the idea conception to the adequate MR design and its subse-quent development.

The tests developed in the MaREMAS prototype were satisfactory, however, we proposes as future work to provide the system a more robust statistical module that allows a better analysis of the research variables and from the results to make useful suggestions to the entrepreneur. Another future work will be to integrate the system with actual market research repositories in order to add more knowledge to the system.

\section{References}

1. Acs, Z. J., Szerb L.: Entrepreneurship, economic growth and public policy. Small Business Economics. 28, 109-122 (2007)

2. Cobo Quesada, F. B., Hervé, A., Sánchez, A., Del Socorro, M.: Emprender en clave de marketing: propuestas conceptuales y prácticas. Anuario jurídico y económico escurialense. 43, 373-392 (2010)

3. González-Benito, Ó., GonzálezBenito, J., Muñoz-Gallego, P. A.: 
Role of entrepreneurship and market orientation in firms' success. European Journal of Marketing. 43, 500-522 (2009)

4. Liu, S. S., Luo, X., Shi, Y. Z.: Marketoriented organizations in an emerging economy: A study of missing links. Journal of Business Research. 56, 481491 (2003)

5. Bolger, F., Pulford, B. D., Colman, A. M.: Market entry decisions: effects of absolute and relative confidence. Exp Psychol. 55, 113-120 (2008)

6. Webb, J. W., Ireland, R. D., Hitt, M. A., Kistruck, G. M., Tihanyi, L.: Where is the opportunity without the customer? An integration of marketing activities, the entrepreneurship process, and institutional theory. Journal of the Academy of Marketing Science. 39, 537-554 (2010)

7. Sciascia, S., Naldi, L., Hunter, E.: Market orientation as determinant of entrepreneurship: an empirical investigation on SMEs. International Entrepreneurship and Management Journal. 2, 21-38 (2006)

8. Wood, M., DeLoach, S.: An overview of the multiagent systems engineering methodology», in Agent-Oriented Software Engineering. 1-53 (2001)

9. Iglesias Fernández, C. A.: Definición de una Metodología para el Desarrollo de Sistemas Multiagente. (1998).

10. Wooldridge, M., Jennings, N. R., Kinny, D.: The GAIA methodology for agent-oriented analysis and design. Autonomous Agents and Multi-Agent Systems. 3, 285-312 (2000)

11. Jiménez, J., Ovalle, D., Bedoya, J. W:: Conceptualización y análisis de un sistema multi-agente pedagógico utilizando la metodología MASCommonKADS. Dyna, 158, 229-239 (2009)

12. Bayer, P., Svantesson, M.: Comparison of Agent-Oriented Methodologies. First Blekinge Institute of Technology Student Work-shop on Agent Programming, 21 (2001)

13. Lázaro Molina, J., Ruiz Dimoff, P.: Apuntes metodológicos de desarrollo orientado a agentes: Aplicación a una agencia de viajes (2006)

14. Alonso Bustos, A.: Implementacion del algoritmo de Computer Assisted Slot Allocation System (CASA) de la Central Flow Management Unit (CFMU). (2011)

15. Pastrana David, M. P., Guzmán, J., Ovalle, D.: Análisis y Diseño de un Sistema Multi-Agente para Simular el Mercado de la energía Eléctrica en Colombia. Universidad Nacional de Colombia, Facultad de Minas - Sede Medellin, (2003)

16. Bellifemine F., Rimessa G., Trucco T. \& Caire G.: JADE Programmer's Guide. (2005) 